\title{
Forensic age prediction for dead or living samples by use of methylation-sensitive high resolution melting
}

\section{AUTHOR(S):}

Hamano, Yuya; Manabe, Sho; Morimoto, Chie; Fujimoto, Shuntaro; Ozeki, Munetaka; Tamaki, Keiji

\section{CITATION:}

Hamano, Yuya ...[et al]. Forensic age prediction for dead or living samples by use of methylation-sensitive high resolution melting. Legal Medicine 2016, 21: 5-10

\section{ISSUE DATE:}

2016-07

URL:

http://hdl.handle.net/2433/216685

\section{RIGHT:}

(c) 2016. This manuscript version is made available under the CC-BY-NC-ND 4.0 license

http://creativecommons.org/licenses/by-nc-nd/4.0/; The full-text file will be made open to the public on $1 \mathrm{July} 2017$ in accordance with publisher's 'Terms and Conditions for Self-Archiving'.; この論文は出版社版でありません。引用の際に は出版社版をご確認ご利用ください。; This is not the published version. Please cite only the published version. 
1 Forensic age prediction for dead or living samples by use of methylation-sensitive high resolution

2

3

4

5

6

7

8

9

Abbreviations: MS-HRM, methylation-sensitive high resolution melting; MAD, mean absolute deviation

\section{melting}

Yuya Hamano, Sho Manabe, Chie Morimoto, Shuntaro Fujimoto, Munetaka Ozeki, Keiji Tamaki

\section{Abstract}

Age prediction with epigenetic information is now edging closer to practical use in forensic community.

Many age-related $\mathrm{CpG}(\mathrm{AR}-\mathrm{CpG})$ sites have proven useful in predicting age in pyrosequencing or DNA chip analyses. In this study, a wide range methylation status in the ELOVL2 and FHL2 promoter regions were detected with methylation-sensitive high resolution melting (MS-HRM) in a labor-, time-, and cost-effective manner. Non-linear-distributions of methylation status and chronological age were newly fitted to the logistic curve. Notably, these distributions were revealed to be similar in 22 living blood samples and 52 dead blood samples. Therefore, the difference of methylation status between living and dead samples suggested to be ignorable by MS-HRM. Additionally, the information from ELOVL2 and FHL2 were integrated into a logistic curve fitting model to develop a final predictive model through the multivariate linear regression of logit-linked methylation rates and chronological age with adjusted $\mathrm{R}^{2}=0.83$. Mean absolute deviation (MAD) was 7.44 for 74 training set and 7.71 for 30 additional independent test set, indicating that the final predicting model is accurate. This suggests that our MS-HRM-based method has great potential in predicting actual forensic age.

Keywords: Age prediction, Forensic science, MS-HRM, DNA methylation 


\section{Introduction}

27

Although age is one of the most important pieces of information for criminal investigations, there are few techniques available to predict age in actual practice, such as examining bones or teeth morphologically. These techniques require expert medical experience, but the result of prediction might not be "objective". Moreover, these are not versatile methods and are limited to samples such as bones or teeth in actual practice. Age-related changes in cytosine methylation have been recently reported by many groups [1-7]. For example, Hannum et al. built a predictive model of aging blood with the use of 71 methylation markers selected from the Illumina Infinium HumanMethylation450 BeadChip, which measures more than 450,000 CpG markers [8]. Branicki et al. investigated the usefulness of CpGs located in the promoter region of ELOVL2 with pyrosequencing $[9,10]$. The promoter region of $F H L 2$ has also been identified as a useful age-predictive marker in many studies $[4,10]$. Owing to these studies, knowledge on the relationships between methylation patterns and chronological age has accumulated. However, the BeadChip method requires specialized instruments and analyzing machines followed by complex bioinformatic analysis for age prediction. The pyrosequencing method also requires specialized instruments. In general, very few forensic laboratories are equipped with these kinds of machines. Even if so, high costs has prevented these methods from being routinely used in criminal investigations.

Methylation-sensitive high resolution melting (MS-HRM) is a method that measures methylation statuses easily, quickly and cost effectively, where bisulfite-treated DNA is PCR amplified followed by melting analysis [11-15]. In bisulfite-treated DNA analyses, unmethylated cytosines are converted to uracil by bisulfite conversion while methylated cytosines are kept intact. Therefore, the information of methylation status is directly converted to the sequence, where it alters the thermodynamic stability of double-stranded DNA, enabling quantitative methylation assessment. The unique characteristic of MS-HRM is that it measures the overall methylation status of amplified PCR products, rather than the individual CpG marker. As a result, the information of many $\mathrm{CpG}$ markers present in the region of interest can be integrated and analyzed with one pair of PCR primers in one measurement.

We have to consider the possibility that post-mortem changes alter the methylation status when performing age prediction in actual cases. For example, a forensic scientist is not always cognizant of whether a victim is alive or deceased, as in abduction cases. Before applying this technology to actual cases, we must investigate the effect of post-mortem changes on forensic age prediction. To our best knowledge, 
no one has focused on this point, which might become a more significant issue when performing age prediction for actual forensic cases.

Here, we report on a labor-, time-, and cost-effective method of forensic age prediction using MS-HRM for the ELOVL2 and FHL2. The analysis of 74 blood samples from 22 living and 52 dead donors who varied in age from 0 to 95 years yielded a logistic curve model. While the majority of previous studies constructed simple linear models for this analysis, such models were not rational for the purposes of our study. Finally, 30 independent dead blood samples were used to test the prediction accuracy of the model.

\section{Materials and methods}

\subsection{Sample collection and DNA extraction}

Blood samples from 19 healthy donors were collected at the same time of health checking. Blood samples from three children were collected from epistaxis caused by daily life hurt rather than performing any operation . For these blood samples, DNA was extracted with QIAamp DNA Investigator Kit (Qiagen, Hilden, Germany) according to the manufacturer's protocol. All donors or their parents signed written consent form prior to donation. Additionally, cadaver blood samples were collected from 82 autopsies performed during 2006-2009 at Kyoto University, Kyoto, Japan. Cadaver blood samples were collected in cases of extrinsic death — such as burn cases or suicides — and all autopsies were performed within 10 days of death. All dead bodies had no evidence of disease (e. g. cancer) which affects the methylation status. DNA from cadaver blood was extracted using the QIAprep DNA Blood Kit and stored at $-20{ }^{\circ} \mathrm{C}$ until use. All samples in this study were used with permission for research use from the ethical committee of Graduate School of Medicine of Kyoto University.

\subsection{Bisulfite modification and control DNA}

All DNA extracted from blood was treated with EpiTect Fast Bisulfite Conversion Kit (Qiagen) and bisulfiteconverted DNA were eluted with Buffer EB (10 mM Tris-Cl, $\mathrm{pH}$ 8.5). The concentration of eluted DNA was then measured with the Nano Vue Plus (GE Healthcare, Amersham, England) and subsequently adjusted to $10 \mathrm{ng} / \mu \mathrm{L}$ with Buffer EB. As a positive (fully methylated) or negative (fully unmethylated) control, we used 
"EpiTect Control DNA (human), methylated/unmethylated and bisulfite converted (Qiagen)" respectively.

Control DNA was stored in Buffer EB and adjusted to $10 \mathrm{ng} / \mu \mathrm{L}$.

88

\subsection{High resolution melting step}

90

PCR primers were designed with BiSearch [16,17] according to Table 1. For ELOVL2, the amplicon is 91 bp long and includes $10 \mathrm{CpG}$ markers between primer binding sites (chr6: 11,044,611-11,044,701; Genome browser UCSC GRCh38, Fig. 1). For FHL 2, the amplicon is 133 bp long and includes 14 CpG markers (chr2: 105,399,228-105,399,360). PCR amplification was carried out with a Roche LightCycler 480 Instrument II (Roche Diagnostics GmbH, Mannheim, Germany) equipped with the Gene Scanning Software (version 1.5.1.62 SP2) in a $25 \mu \mathrm{L}$ total volume containing: 1x EpiTect HRM PCR Master Mix (EpiTect HRM PCR Kit, Qiagen), $250 \mathrm{nM}$ of each primer and $20 \mathrm{ng}$ of bisulfite modified template. First, polymerase was activated at $95{ }^{\circ} \mathrm{C}$ for $5 \mathrm{~min}$, followed by 45 cycles of $95{ }^{\circ} \mathrm{C}$ for $10 \mathrm{~s}, 50^{\circ} \mathrm{C}$ for $30 \mathrm{~s}$, and $72{ }^{\circ} \mathrm{C}$ for $10 \mathrm{~s}$. After the amplification, HRM analysis was initiated by denaturing all products at $95{ }^{\circ} \mathrm{C}$ for $1 \mathrm{~min}$, followed by re-annealing at $40{ }^{\circ} \mathrm{C}$ for $1 \mathrm{~min}$. Subsequently, the samples were quickly warmed to $50{ }^{\circ} \mathrm{C}$ and heated to $95{ }^{\circ} \mathrm{C}$ at $0.1{ }^{\circ} \mathrm{C} / \mathrm{s}$. Fluorescence intensity was measured at 25 acquisitions/s. All reactions were performed in duplicate.

When HRM analysis was performed, Gene Scanning Software first normalized raw melt curves so that different samples can be compared. In this normalizing process, we set the pre-melt temperature region to $68-69^{\circ} \mathrm{C}$ and the post-melt temperature region to $82-83{ }^{\circ} \mathrm{C}$. Although, the temperature shift process is often run when the software is used for analyzing heterozygous mutant, no adjustment was performed in this study by setting the threshold to zero, because the shape of melt curve itself was important in analysis of the overall methylation status of the amplicon. If the temperature shift process was performed, the shape of melt curve would be distorted. A difference curve was then derived from the first derivative of the melt curves, after setting the data of fully unmethylated sample as a baseline. Relative signal difference values were exported as .txt data, and the maximum absolute value were defined as "Df value" for each sample (Fig. 2B). 
116 pair rich sequence after bisulfite modification) tends to be amplified more efficiently than methylated DNA

117 (CG pair rich sequence). Therefore, a standard curve was first established for each target site to accurately

118 measure methylation status. Fully methylated control DNA and fully unmethylated DNA were mixed in 119 appropriate ratios to make $0 \%, 25 \%, 50 \%, 65 \%, 80 \%, 90 \%$, and $100 \%$ methylated control DNA. For the 90\% and 100\% methylated standard sample of ELOVL2, $40 \mathrm{ng}$ bisulfite-treated DNA was used as a template due to its small amplification efficiency caused by PCR bias. Df values of each control sample were plotted and a non-linear regression model was developed [18] with R (version 3.2.2) [20] depicted as Equation 1 as follows

$$
\frac{\mathrm{a} * \mathrm{M}}{100-\mathrm{M}}=\frac{\mathrm{Df}}{\mathrm{Df}_{\max }-\mathrm{Df}}
$$

where $\mathrm{M}$ is the proportion of a methylation status and $\mathrm{Df}_{\max }$ is the $\mathrm{Df}$ value of $100 \%$ methylated control sample and " $\mathrm{a}$ " is a coefficient. Once the standard curve is established, the overall methylation status of the sample can be calculated by substituting the Df value to the Eq. (1). Therefore, Df values can be converted to methylation status.

\subsection{Statistical Analysis}

First, logistic curve fittings were performed with $\mathrm{R}$ to determine the relationship between age and methylation status. In this fitting, the value of methylation status is converted to the logit form, and it was fitted to a line by ordinary least squares depicted as Equation 2 for ELOVL2 and FHL2 each.

$$
\text { Predicted age }=b+c * \ln \frac{M}{1-M}
$$

where "b, c" are coefficients. Secondly, ANCOVA were performed with IBM SPSS Statistics 20 to confirm whether live status (dead or alive) affects the regression line or not $(p<0.05$ is considered as statistically significant). 


$$
\text { Predicted age }=d+e * \ln \frac{M_{E}}{1-M_{E}}+f * \ln \frac{M_{F}}{1-M_{F}}
$$

Where $\mathrm{M}_{\mathrm{E}}$ and $\mathrm{M}_{\mathrm{F}}$ stand for the proportion of a methylation status of ELOVL2 and FHL2, respectively, and

149 “d, e, f" are coefficients. The prediction accuracy of the regression model was assessed using the adjusted $\mathrm{R}^{2}$. The mean absolute deviation (MAD) was also calculated. The final model was further validated using an additional set of 30 test samples (all dead).

\section{Results}

\subsection{Methylation analysis with MS-HRM}

Smooth melting curves were obtained from MS-HRM (Fig. 2A) and difference curves were also obtained with Gene Scanning Software using the $0 \%$ methylated data as a baseline (Fig. 2B). Table 2 shows the estimated "a" value of the Eq. (1) for ELOVL2 and FHL2, meaning that unmethylated DNA exhibits a 5-fold or 5.6-fold amplification efficiently in MS-HRM due to PCR bias (Fig. 2C). In this study, methylation rate differences between $40 \%-100 \%$ can be detected clearly by the differences of Df values both for ELOVL2 and $F H L 2$, while those between $0 \%-30 \%$ were hard to detect.

163

\subsection{Assessment of the methylation status difference between living and dead}

Fig. 3A, D shows the methylation status of ELOVL2 and FHL2 for 74 training samples (22 alive; 52 dead). Coefficient values of the Eq. (2) ("b", "c") are listed in Table 2. Chronological age and logit-linked methylation status correlated well. Therefore, we decided to adopt logistic curve model for predicting age, while simple linear model is adopted in most of researches performed in past. Two simple linear regression lines of logit-linked methylation status and age derived from living or dead sample has no statistically significant difference $(\mathrm{p} \geq 0.05)$ in slope and intercept for each target site (Fig. 3 B, E). New regression models explained nearly $80 \%$ of the variation in age when combining living samples and dead samples (Fig. 
$1733 \mathrm{C}$, F; adjusted $\mathrm{R}^{2}$ was 0.74 for $E L O V L 2$ and 0.81 for $F H L 2$ ). Negative prediction values obtained for three

174 or four young individuals were set at $0[9,10]$. The mean absolute deviation (MAD) was 9.67 for ELOVL2

175 and 7.71 for FHL2. The prediction accuracy was good for the youth but a little poor for the old. No statistically significant difference in slope and intercept were observed based on gender either (Fig. S1). Thus, we decided to ignore differences due to gender to keep the prediction model simple.

\subsection{Developing final age prediction model and its validation}

The final age prediction model was developed combining the methylation information of ELOVL2 and FHL2. Estimated coefficient values of Eq. (3) (“d”, "e", "f”) are also listed in Table 2. This multivariate regression model showed further accuracy with MAD 7.44 (Fig. 4). In the end, holdout validation test was performed. The methylation status of 30 additional independent samples was analyzed and applied for the final model. The result is also shown in Fig. 4. MAD was slightly higher in this test group (7.71).

\section{Discussion}

Age prediction with the epigenetic techniques has attracted increasing attention from the forensic science community. For investigation of crimes, it is important to minimize the time required to obtain test results. In this study, MS-HRM was adopted to analyze the methylation status of the ELOVL2 and FHL2 promoter regions, which is able to return a test result within half a day after blood sample acquisition. The unique characteristic of this method is that it can detect the overall methylation status of the region of interest. Branicki et al. found that the methylation rates of $\mathrm{CpG}$ sites near $\mathrm{AR}-\mathrm{CpG}$ correlate well with chronological age for ELOVL2 (C1-C7 in his study [9]). The methylation status of many CpG sites can be detected with only one MS-HRM analysis, while DNA chip can detect only a limited number of CpG sites. On the other hand, MS-HRM has its limitations for practical usage. The biggest limitation might be the issue of PCR bias $[18,19]$, where methylated templates (containing many Cs in the sequence) are less effectively amplified than unmethylated templates (contain many Us). Owing to the PCR bias, the methylation status differences are hard to detect in lower methylated region (e.g. 0\%-30\%). Therefore, if a researcher wanted to detect differences in the lowly methylated region with MS-HRM, he/she would have to identify a sequence without any PCR bias or design primers that reverse the PCR bias [19]. As for this study, PCR bias does not affect 
the detectability of methylation differences across samples for analyzing higher methylated regions. In addition, if $\mathrm{CpG}$ sites in a $\mathrm{CpG}$ island were intended to be analyzed, designing primers might be a little difficult because they should include as little $\mathrm{CpG}$ sites as possible.

The speed of the methylation change with aging has been less discussed. Most researchers have developed simple linear regression models for the methylation status and age (i.e. the speed of methylation change is constant through one's lifetime); however, these models have not accounted for pediatric specimens. Alisch et al. pointed out that the methylation changes accelerate in childhood for some $\mathrm{CpG}$ sites [21]. We found that changes of methylation status in the promoter region of ELOVL2 and FHL2 were not linear, but rather increased dramatically in youth and slowed down with increasing age. The methylation change for two target sites could be fitted well with a logistic or growth curve (adjusted $\mathrm{R}^{2}=0.74$ and 0.81 ). It is reasonable to hypothesize that the methylation status plateaus with increasing age than to hypothesize that it has no upper limit.

The prediction accuracy depended on sample donor's chronological age in this study (Fig. 4), which was concordant with the research performed by Branicki et al. Age estimation for youths ( $0-20$ years old) had little prediction error, but this error increased with chronological age. This might be explained by individual differences in the rate of methylation change. At first, individual differences may be slight, but they accumulate with age. Therefore, a prediction result must be handled carefully when the predicted age is high ( $>50$ years old). Similarly, it is highly reliable when the predicted age is low ( $0-20$ years old).

To our best knowledge, it is unknown whether death affects the methylation status. If post-mortem changes affect one's methylation status, forensic researchers cannot predict age without information about the sample donor's safety. In this study, all blood samples from dead bodies were collected within 10 days after death and the methylation status was analyzed. The distribution of age and methylation rate was similar regardless of the sample donor's life or death (Fig. 3 A,D). Moreover, no statistically significant change was observed between living and dead blood samples. These observations may suggest that the difference of methylation status between living and dead samples is ignorable, though sample size is limited. It was difficult to collect more living blood samples especially for youths. Further analysis might be required to support our findings.

Additionally, differences in methylation status due to gender were considered (Fig. S1). No statistically significant change was observed between male and female genders, which is consistent with findings of other studies. Huang et al. developed an age prediction model for the gender combined case and two 
additional models for only male or only female cases; however, no statistically significant difference was

234 observed between the gender-based models [6]. Branicki et al. decided not to include gender in prediction modeling ( $\mathrm{R}^{2}$ improved by 0.001 with age as a covariate) [10]. In our study, age prediction accuracy was minimally affected by distinguishing gender, and therefore, the simplicity of the combined model was preferred.

The multivariate regression model enhanced the prediction accuracy when combining the information about the methylation status of ELOVL2 and FHL2 in our study. It is well known that increasing the number of target sites enhances age prediction accuracy-Weidner et al. developed a prediction model with 3 target genes $(\mathrm{MAD}=5.4)$, while more accurate model required 102 sites $(\mathrm{MAD}=3.34)$ [2]. Additional target sites may also increase the prediction accuracy of our method. The authors are investigating other target sites with MS-HRM in order to improve the prediction accuracy now. However, analyzing more than 5 target sites might be too labor-intensive to perform age prediction in actual forensic cases.

Age prediction with epigenetic information has become popular in the forensic science community. This knowledge should now be used for actual criminal investigations. Most previous reports analyzed the methylation rate of $\mathrm{CpG}$ sites by using DNA chips or pyrosequencing. However, these techniques are too labor-, time-, and cost-intensive to apply to routine crime investigations. MS-HRM has the potential to be a gold standard for usual forensic test because of its convenience. However, there are a few causes for caution before applying this method to actual cases. First, the standard curve of methylation rate and Df value must be determined for each MS-HRM instrument and chemical because commercially available fully unmethylated DNA may not be perfectly unmethylated. Second, methylation analysis should be performed more than twice to validate the obtained data. Third, test samples are limited to blood samples. AR-CpG is considered to differ by tissue $[3,5,7]$. We are attempting to develop an age prediction model using saliva, semen, sweat, and bone samples with MS-HRM, but only the blood model has been fully developed. Huang et al. have shown that there was no statistically significant difference in age prediction results from blood samples and those from bloodstain [6]. When these points are considered, forensic age prediction for dead or living samples can be performed with MS-HRM conveniently. For instance, analysis of a bloodstain left at a crime scene could provide the approximate age of the suspect or victim. Thus, we believe that this study opens new possibilities for forensic DNA phenotyping. 
264 The authors would like to offer special thanks to Prof. Ryosuke Takahashi (Department of Neurology,

265 Graduate School of Medicine, Kyoto University) for his technical support. We also thank Dr. Ken Watanabe

266 (National Research Institute of Police Science, NRIPS) whose comments made an enormous contribution to 267 this work.

268

269 Role of Funding Source

270

271

This study was supported by the operation grants of Department of Forensic Medicine, Graduate School of 272 Medicine, Kyoto University.

273

\section{Conflict of Interest}

275

276 The authors state that they have no conflicts of interest.

277 
280 [1] P. Garagnani, M.G. Bacalini, C. Pirazzini, D. Gori, C. Giuliani, D. Mari, A.M. Di Blasio, D. Gentilini, gene as a new epigenetic marker of age. Aging Cell 2012;11:1132-1134.

[2] C.I. Weidner, Q. Lin, C.M. Koch, L. Eisele, F. Beier, P. Ziegler, D.O. Bauerschlag, K.H. Jöckel, R. Erbel, T.W. Mühleisen, M. Zenke, T.H. Brümmendorf, W. Wagner. Aging of blood can be tracked by DNA methylation changes at just three CpG sites. Genome Biol. 2014;15:R24.

[3] K. Day, L.L. Waite, A. Thalacker-Mercer, A. West, M.M. Bamman, J.D. Brooks, R.M. Myers, D. Absher.

Differential DNA methylation with age displays both common and dynamic features across human tissues that are influenced by CpG landscape. Genome Biol. 2013;14:R102.

[4] I. Florath, K. Butterbach, H. Müller, M. Bewerunge-Hudler, H. Brenner. Cross-sectional and longitudinal changes in DNA methylation with age: an epigenome-wide analysis revealing over 60 novel age-associated CpG sites. Hum. Mol. Genet. 2014;23:1186-1201.

[5] S. Horvath. DNA methylation age of human tissues and cell types. Genome Biol. 2013;14:R115.

[6] Y. Huang, J. Yan, J. Hou, X. Fu, L. Li, Y. Hou. Developing a DNA methylation assay for human age prediction in blood and bloodstain. Forensic Sci. Int. Genet. 2015;17:129-136. [7] H. Y. Lee, Sang-Eun Jung, Y. N. Oh, A. Choi, W. I. Yang, Kyoung-Jin Shin. Epigenetic age signatures in the forensically relevant body fluid of semen : a preliminary study. Forensic Sci. Int. Genet. 2015;19:28-34. [8] G. Hannum, J. Guinney, L. Zhao, L. Zhang, G. Hughes, S. Sadda, B. Klotzle, M. Bibikova, J.B. Fan, Y. Gao, R. Deconde, M. Chen, I. Rajapakse, S. Friend, T. Ideker, K. Zhang. Genome-wide methylation profiles reveal quantitative views of human aging rates. Mol. Cell 2013;49:359-367.

R. Płoski, W. Branicki. Examination of DNA methylation status of the ELOVL2 marker may be useful for human age prediction in forensic science. Forensic Sci. Int. Genet. 2015;14:161-167.

[10] R. Zbieć-Piekarska, M. Spólnicka, T Kupiec, A. Parys-Proszek, Ż Makowska, A. Pałeczka, K.

Kucharczyk, R. Płoski, W. Branicki. Development of a forensically useful age prediction method based on DNA methylation analysis. Forensic Sci. Int. Genet. 2015;17:173-179.

[11] T.K. Wojdacz, A. Dobrovic, L.L. Hansen. Methylation-sensitive high-resolution melting. Nat. Protoc. 2008;3:1903-1908. 
308 [12] T.K. Wojdacz, A. Dobrovic. Methylation-sensitive high resolution melting (MS-HRM): a new approach 309 for sensitive and high-throughput assessment of methylation. Nucleic Acids Res. 2007;35:e41.

310 [13] M.Y. Tse, J.E. Ashbury, N. Zwingerman, W.D. King, S.A. Taylor, S.C. Pang. A refined, rapid and 311 reproducible high resolution melt (HRM)-based method suitable for quantification of global LINE-1 312 repetitive element methylation. BMC Res. Notes 2011;4:565.

313 [14] H.G. Hernandez, M.Y. Tse, S.C. Pang, H. Arboleda, D.A. Forero. Optimizing methodologies for PCR314 based DNA methylation analysis. Biotechniques 2013;55:181-197.

315 [15] L. Stewart, N Evans, K.J. Bexon, D.J. van der Meer, G.A. Williams. Differentiating between 316 monozygotic twins through DNA methylation-specific high-resolution melt curve analysis. Anal. Biochem. $317 \quad 2015 ; 476: 36-39$

318 [16] G.E. Tusnady, I. Simon, A. Varadi, T. Aranyi. BiSearch: primer-design and search tool for PCR on 319 bisulfite-treated genomes. Nucleic Acids Res. 2005;33:e9.

320 [17] T. Aranyi, A. Varadi, I. Simon, G.E. Tusnady. The Bisearch web server. BMC Bioinformatics $321 \quad 2006 ; 7: 431$.

322 [18] P.M. Warnecka, C. Stirzaker, J.R. Melki, D.S. Millar, C.L. Paul, S.J. Clark. Detection and measurement 323 of PCR bias in quantitative methylation analysis of bisulphite-treated DNA. Nucleic Acids Res. $324 \quad 1997 ; 25: 4422-4426$.

325 [19] T.K. Wojdacz, L.L. Hansen. Reversal of PCR bias for improved sensitivity of the DNA methylation 326 melting curve assay. Biotechniques 2006;41:274-278.

327 [20] R Development Core Team. R: a language and environment for statistical computing. Vienna. Austria: 328 R Foundation for Statistical Computing. 2006. Available from: http://www.R-project.org.

329 [21] R.S. Alisch, B.G. Barwick, P. Chopra. Age-associated DNA methylation in pediatric populations. $330 \quad$ Genome Res. 2012;22:623-632. 


\section{$332 \quad$ Table 1}

333 The sequence of PCR primers

\begin{tabular}{ll}
\hline Primers & Primer sequence (5' to 3 ') \\
\hline ELOVL2-Fw & CGATTTGTAGGTTTAGT \\
$E L O V L 2-\mathrm{Rv}$ & ACTACCAATCTAAACAA \\
FHL2-Fw & TTTACCAAAACTCCTTTCTT \\
FHL2-Rv & GTGGGTAGATTTTTGTTATT \\
\hline
\end{tabular}

334

335

336 Table 2

337 Coefficients calculated in this study

\begin{tabular}{lcccccc}
\hline & $\mathrm{a}\left(\times 10^{-2}\right)$ & $\mathrm{b}$ & $\mathrm{c}$ & $\mathrm{d}$ & $\mathrm{e}$ & $\mathrm{f}$ \\
\hline ELOVL2 & 19.7 & -28.8 & 22.9 & & & \\
FHL2 & 9.8 & -21.1 & 45.1 & & & \\
Combined & & & & -37.2 & 14.6 & 18.9 \\
\hline
\end{tabular}

338

339 


\section{$340 \quad$ Figure captions}

341

342 Fig. 1. Sequences of PCR target sites in this study (before bisulfite conversion). PCR primer binding sites

343 are boxed. CpG markers that can be analyzed by MS-HRM are emphasized and underlined.

345 Fig. 2. A) Representative normalized melting data. Melting data of $0 \%$ methylated standard sample is

346 highlighted with light blue. B) Difference curves obtained by Gene Scanning software. The 0\% methylated 347 standard sample was set as a baseline. C) Standard curves of the relationship between methylation rates and Df value for $0 \%, 25 \%, 50 \%, 65 \%, 80 \%, 90 \%$, and $100 \%$ methylated standard samples.

Fig. 3. A, D) Distribution of methylation status and chronological age for living and dead blood samples. prediction. Negative prediction values were set at 0 .

Fig. 4. Accuracy of the final age predicting model with combined information of the methylation status of ELOVL2 and FHL2 for 74 training set and 30 test set.

Fig. S1. Relationship between chronological age and logit-linked methylation status for males or females. 
359 Fig. 1.

ELOVL2

...GCGG

CGATTTGCAGGTCCAGACGGCGCCGGTTTCGCGCG

GCGGCTCAACGTCCACGGAGCCCCAGGAATACCCA CCCGCTGCCCAGATCGGCAGCCGCT...

FHL2

...TTG

TTTGCCAGGGCTCCTTTCTTCGTGCCCTCCGGGTC

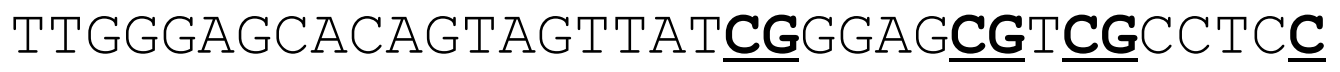

GGCGTGGGCTCTEGGGCGCGAGTTTEGGACGAGGC

CTGGGCGCGGTGGCAGGGGTCTGCCCACGCC... 
$361 \quad$ Fig. 2.
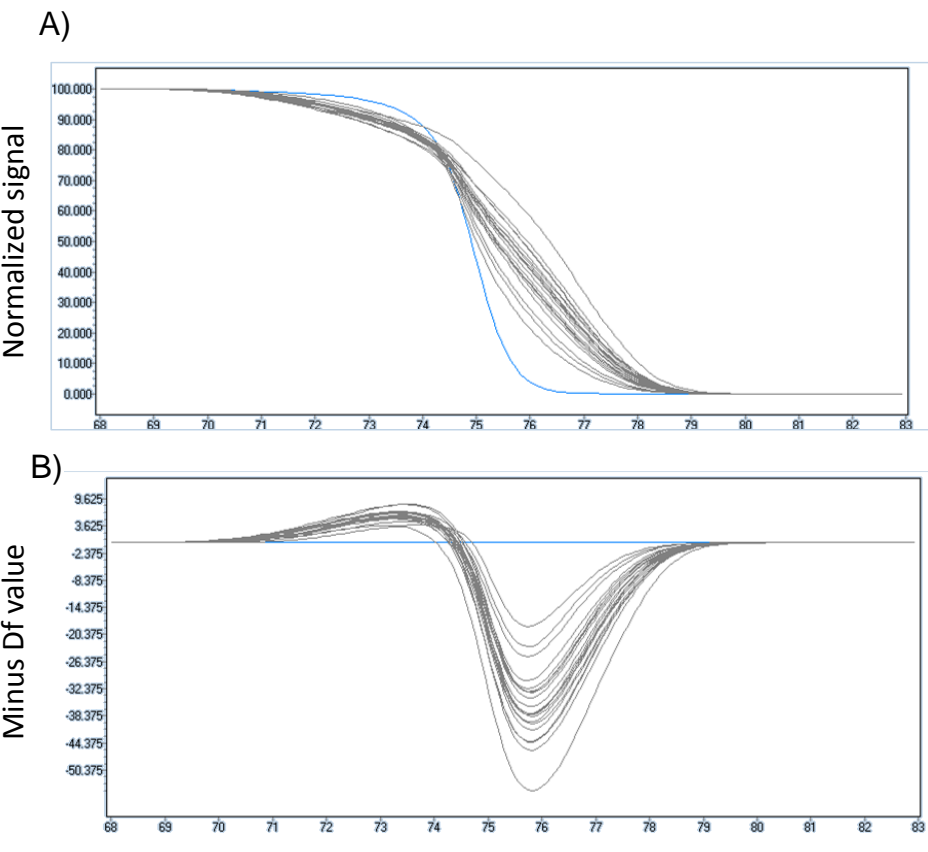

C)

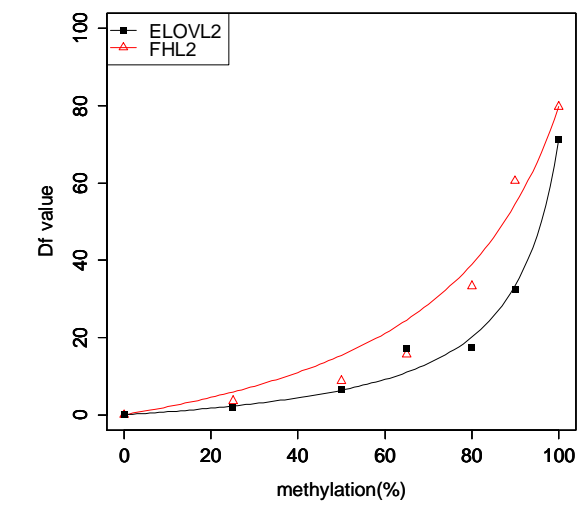

362

Temperature

363 
$364 \quad$ Fig. 3

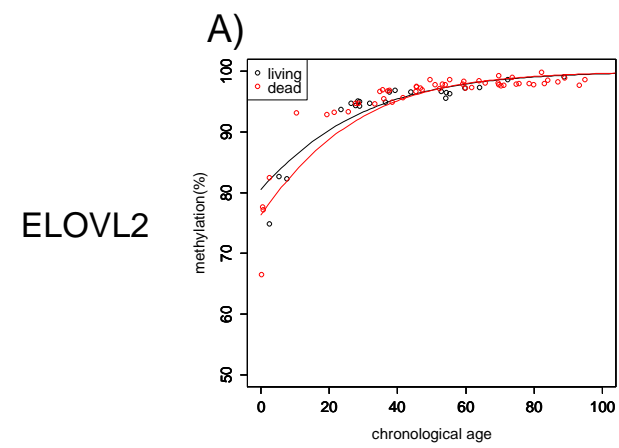

B)

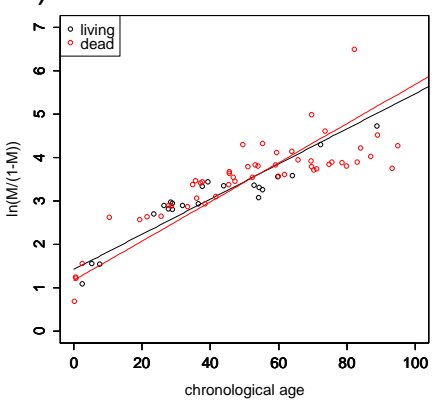

D)

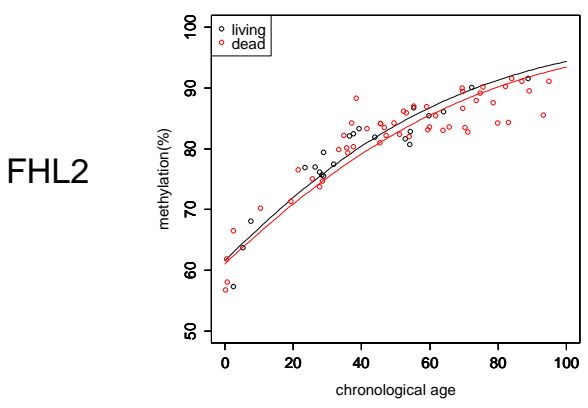

E)

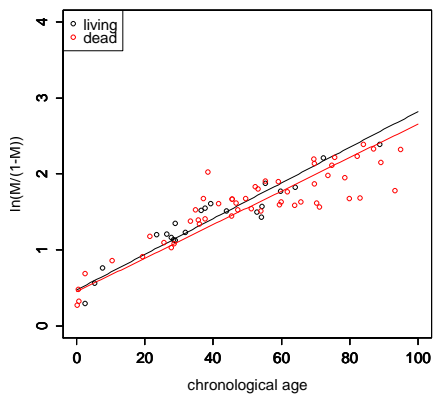

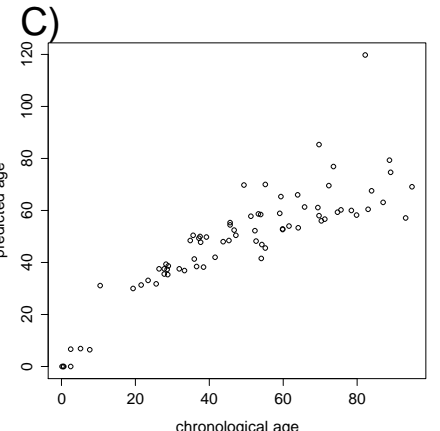

F)

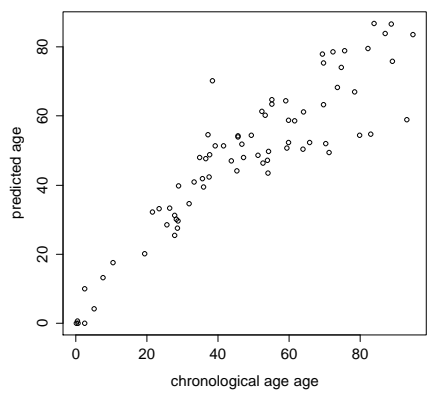


$367 \quad$ Fig. 4

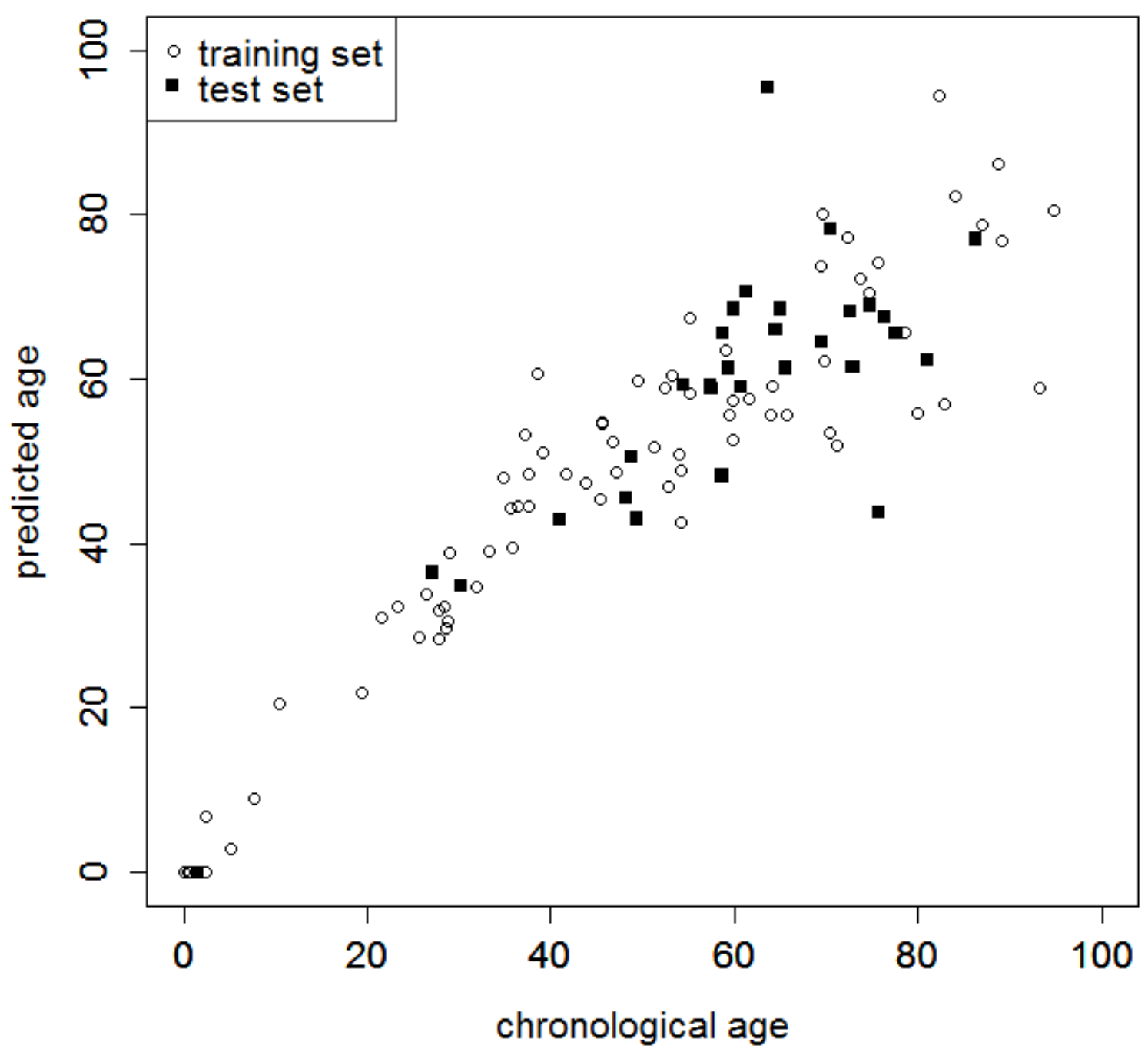

368 
$370 \quad$ Fig. S1

ELOVL2

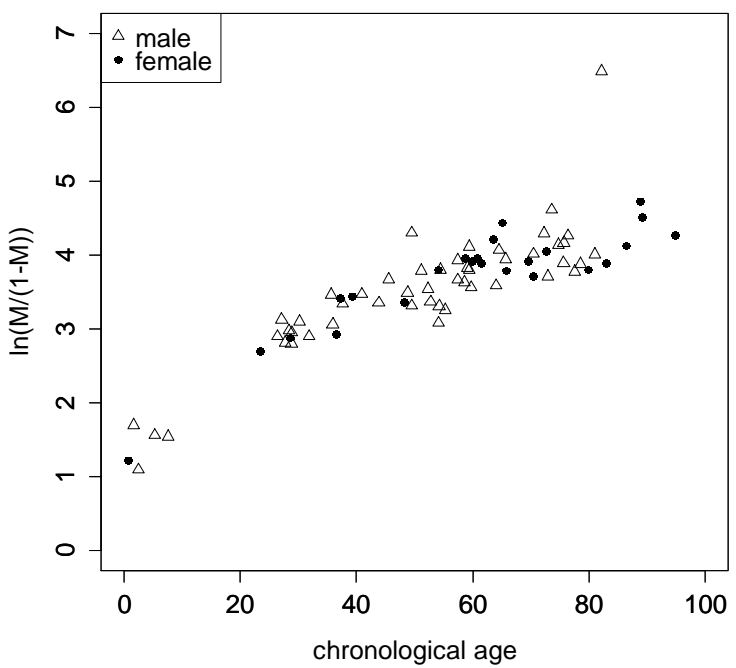

FHL2

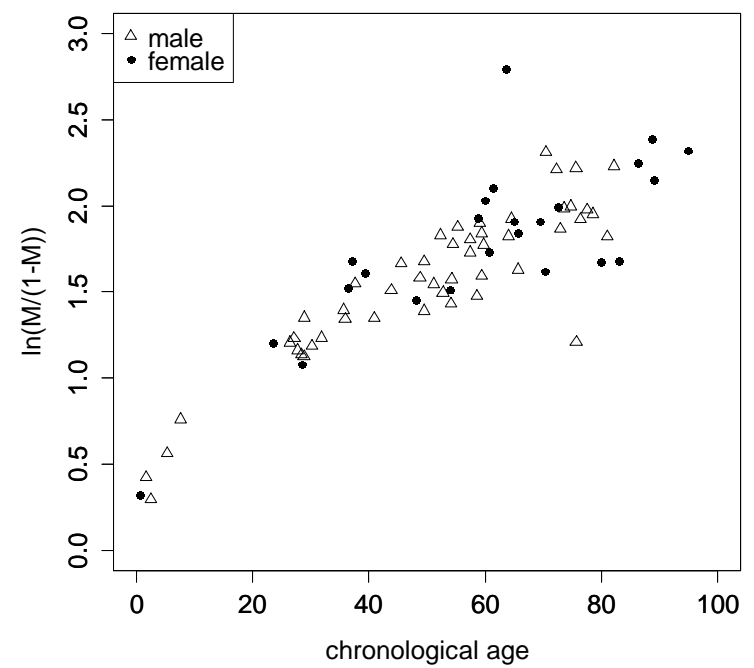

